\title{
Histopathological analysis of the inflammatory response of two invasive techniques in the calcaneal tendon of a mouse
}

\author{
Medina i Mirapeix F. ${ }^{1}$ García Vidal J.A. ${ }^{1}$ Escolar Reina P. ${ }^{1}$ Martínez Cáceres C.M. ${ }^{2}$ \\ ${ }^{1}$ Department of Physiotherapy. Universidad de Murcia, Murcia, Spain \\ 2 Servicio de Patologia. Instituto Murciano de Investigacion \\ Biosanitaria (IMIB), Murcia, Spain
}

Rev Fisioter Invasiva 2019;2:91.

\begin{abstract}
Keywords

- tendon

- mouse

- percutaneous needle electrolysis

- inflammation

Background and Aim Percutaneous needle electrolysis (PNE) is a therapeutic tool which has demonstrated its effectiveness of the treatment of tendinopathies and muscle problems. Previous authors have based the therapeutic effect of the same on the ability to provoke an important inflammatory response. However, there is a scarcity of basic research directed at understanding its effects on a cellular and histological level. The aim of this study was to histopathologically compare the inflammatory response provoked by PNE versus dry needling (DN) in a healthy animal model.

Material and Methods For the proposed study aim a murine model was used with a total of $18 \mathrm{C} 57 \mathrm{BL} / 6 \mathrm{~J}$ mice. All animals received an application of DN in the left common calcaneal tendon, whereas on the right, treatment with PNE was applied ( 3 impacts of $3 \mathrm{~mA}$ during 3 seconds). Groups of animals were sacrificed at 3, 7 and 15 days to evaluate the histopathological evolution of the lesions. Samples of the common calcaneal tendon were taken and fixed in commercial formalin at $4 \%$ tamponed during 24 hours, processed and included in paraffin blocks. Sections of 3 microns thick were performed which were dyed with hematoxylin-eosin for conventional histopathological examination.

Results Dry needling of the common calcaneal tendon induced the presence of an acute inflammatory infiltrate (characterized by the presence of polymorphonuclear neutrophils and macrophages) in the peritenon and adjacent fat tissue which was detectable after the third day, and which was most notable on days 7 and 14 . The application of PNE induced a pattern with a similar behavior, however on day 14 , the inflammatory infiltrate of PNE was more evident compared with DN.

Conclusion The proposed in vivo murine model has been useful for the study of the first phases of the regeneration process induced by both techniques. The histopathological results show that PNE generates a superior stimulus compared to DN on day 14 .
\end{abstract}

DOI https://doi.org/ 10.1055/s-0039-3401870. ISSN 2386-4591.
Copyright (c) 2019 by Thieme Revinter Publicações Ltda, Rio de Janeiro, Brazil 